\title{
MODULATION OF CONTINUOUS ELECTRON BEAMS IN PLASMA WAKE-FIELDS
}

\author{
J. B. ROSENZWEIG
}

DE90 008708

High Energy Physics Division

Argonne National Laboratory

Argonne, Ilinois 60499

\begin{abstract}
In this paper we discuss the interaction of a continuous electron beam with wake-field generated plasma waves. Using a one-dimensional two fluid model, a fully nonlinear analytical description of the interaction is obtained. The phenomena of continuous beam modulation and wave period shortening are discussed. The relationship between these effects and the two-stream instability is also examined.

\section{DISCLAIMER}

\begin{abstract}
This report was prepared as an account of work sponsored by an agency of the United States Government. Neither the United States Government nor any agency thereol, nor any of their employees, makes any warranty, express or implied, or assumes any legal liability or responsibility for the accuracy, completeness, or usefulness of any information, apparatus, product, or process disclosed, or represents that its use would not infringe privately owned rights. Refe:ence herein to any specific commercial product, process, or service by trade name, trademark, manufacturer, or otherwise does not necessarily constitute or imply its endorsement, recom. mendation, or favoring by the United States Government or any agency thereof. The views and opinions of authors expressed herein do not necessarily state or reflect those of the United States Government or any agency thereof.
\end{abstract}

\footnotetext{
Work supported by the U.S. Department of Energy, Division of
} High Energy Physics, Contract W-3I-109-ENG-38.

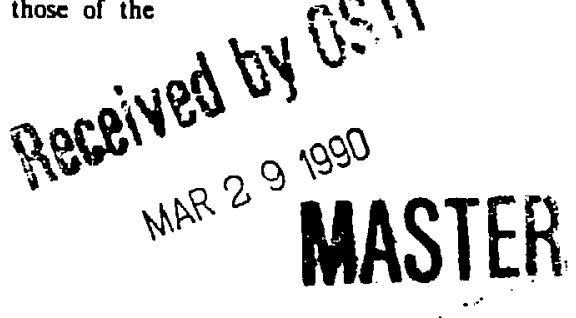


The Plasma Wake-field Accelerator (PWFA), proposed by Chen, Dawson, Huff and Katsouleas ${ }^{\prime \prime \prime}$ in 1984, uses electrostatic fields generated in a plasma wave driven by a relativistic electron beam. Charged particles can be accelerated in this electrostatic wake to ultra-high energies. The linear theory of the PWFA is well developed, and extensive analytical and numerical exist treatments of such issues as transformer ratio (the ratio of maximum accelerating field behind the driving beam to the maximum decelerating field inside the driving beam) and beam loading of the plasma waves. ${ }^{[2,3]}$ Experimental observation, at the ANL Advanced Accelerator Test Facility, of acceleration by the PWFA mechanism in both linear and nonlinear regimes has been recently reported. ${ }^{|3|}$

Aithough the experiments performed in the nonlinear regime of the PWFA displayed interesting three-dimensional effects, ${ }^{[4]}$ only one-dimensional treatments of nonlinear plasma wake-fields currently exist. This problem has been treated by Rosenzweig, ${ }^{[0]}$ Ruth et al. ${ }^{[7]}$ and Amatuni et al., ${ }^{[0]}$ and these investigations predict certain advantages of the nonlinear regime over the linear scheme. Operation in the one-dimensional limit of the non inear regime allows a novel, straightforward method of transformer ratio enhancement, and lowers the plasma density needed to achieve high accelerating gradients in the wake-fields. There are no theoretical predictions as yet on the effects of three-dimensional plasma dynamics on this scheme.

The high transformer ratios obtained in the nonlinear scheme depend on driving the plasma electron density waves to a extremely large amplitudes. For this reason, the maximum wave amplitude, which is limited by trapping of thermal plasma electrons, has been investigated in detail. ${ }^{10,10 \mid}$ For cool laboratory plasmas 
$\left(k T_{e} \simeq 10 \mathrm{eV}\right)$, the transformer ratio is limited to approximately ten by thermal considerations. In Ref. 9 the motion of charged particles in the plasma wakefields is treated generally, to include both the trapping of background plasma electrons as they are overtaken by the driving beam and the motion of particles externally injected into the wake-fields.

Both trapping and injection of charged particles into the plasma waves imply beam loading of the wave's accelerating field. This problem is treated in the case of rigidly positioned beam charge distributions in Ref. 3 for the linear case and Ref. 6 for the nonlinear case, and general solutions for the wake-fields in the presence of beam loading are obtained. The self-consistent motion of the beam charge distributions is ignored in these treatments. This Letter provides a bridge between the two approaches, by calculating the self-consistent effects of beam loading and equations of motion simultaneously for a continuous, lower energy electron beam as it is overtaken by an ultra-relativistic driving beam in a plasma. We also discuss the relationship of the two-stream instability to this result, and examine the potential use of this instability for amplification of plasma wake-field amplitudes.

We begin our treatment by writing, in the spirit of Refs. 6 - 10, the cold fluid equations for two fluids: the plasma electrons, and the continuous beam electrons. of our treatment is the equations of cold nonrelativistic nonlinear fluid theory. We assume that the plasma ions form an immobile neutralizing background of density $n_{0}$ and define the plasma electron density $n$, velocity $v=\beta c$, and the plasma frequency $\omega_{p}=\sqrt{4 \pi e^{2} n_{0} / m_{e}}$ ). The continuous beam and velocity are symbolized by $n_{c}$ and $v_{c}=\beta_{c} c$, and ultra-relativistic bunched beam densities 
and velocities (which are taken to be constant) by $n_{b}$ and $\mathbf{v}_{\mathbf{b}}=\beta_{\mathbf{b}} c$. The fluid equations are thus

$$
\begin{gathered}
\nabla \cdot \mathbf{E}=4 \pi e\left(n_{0}-n-n_{b}-n_{c}\right), \\
\nabla \times \mathbf{E}+\frac{1}{c} \frac{\partial \mathbf{B}}{\partial t}=0 \\
\nabla \times \mathbf{B}=\frac{1}{c} \frac{\partial \mathbf{E}}{\partial t}-4 \pi e\left(n \beta+n_{b} \beta_{\mathbf{b}}+n_{c} \beta_{c}\right), \\
\nabla \cdot \mathbf{B}=0 \\
\frac{\partial \mathbf{p}}{\partial t}+\mathbf{v} \cdot \nabla \mathbf{p}=-e(\mathbf{E}+\beta \times \mathbf{B}),
\end{gathered}
$$

where for the plasma electron fluid motion $p=m_{e} v / \sqrt{1-\beta^{2}}$. The analogous equation of motion for the continuous beam fluid is obtained by substituting $\mathbf{v}_{c}$ for $\mathbf{v}$ in Eq. (5).

For the one-dimensional treatment we take the direction of beam propagation to be $z$, and assume that there is no transverse motion. Additionally we assume the wave motion is a function only of the variable $\tau=\omega_{p}\left(t-z / v_{b}\right)$, and, taking the limit that driving bunched beam (and thus the wave phase velocity) $v_{b} \rightarrow c$, we obtain nonlinear differential equations for the fluid motion. Assuming total charge and current neutrality before the arrival of the bunched beam, and writing the initial unperturbed continuous beam density and velocity as $n_{c 0}$ and $v_{c 0}=\beta_{c 0}$ 
we have from Eqs. (1) and (3)

$$
\frac{n}{n_{0}}=\frac{1+\epsilon\left(\beta_{c 0}-1\right)}{1-\beta}
$$

and

$$
\frac{n_{c}}{n_{0}}=\epsilon\left[\frac{\beta_{c 0}-1}{\beta_{c}-1}\right]
$$

where $\epsilon=n_{\mathrm{eO}} / n_{0}$. Using the dependent variables $x=[(1-\beta) /(1+\beta)]^{1 / 2}$ and $x_{c}=\left[\left(1-\beta_{c}\right) /\left(1+\beta_{c}\right)\right]^{1 / 2}$ the fluid equations derived from Eqs. (5) - (4) reduce to one expression

$$
x^{\prime \prime}=x_{c}^{\prime \prime}=\frac{1}{2}\left[2 \alpha+\frac{1}{x^{2}}-1+\epsilon\left(1-\beta_{c 0}\right)\left(\frac{1}{x^{2}}-\frac{1}{x_{c}^{2}}\right)\right]
$$

where the constant $\alpha=n_{b} / n_{0}$. This expression can be understood physically by noting that are $x$ and $x_{c}$ are proportional to the electrostatic potential $\left.\right|^{|c|}$ and that Eq. (8) is equivalent to the Poisson equation. Thus $x_{c}=x-\lambda$, where the constant $\lambda$ is evaluated from the initial conditions to be

$$
\lambda=\left[\frac{1+\epsilon\left(1-\beta_{\mathrm{co}}\right.}{1-\epsilon\left(1+\beta_{\mathrm{c} 0}\right)}\right]^{1 / 2}-\left[\frac{1-\beta_{\mathrm{c} 0}}{1+\beta_{\mathrm{c} 0}}\right]^{1 / 2}
$$

and the fluid equations have been reduced to a single elliptical equation. Upon substitution of Eq. (9) we obtain the first integral of Eq. (8)

$$
\left(x^{\prime}\right)^{2}=C-\left[2 \alpha+x+\frac{1}{x}+\epsilon\left(1-\beta_{c 0}\right)\left(\frac{1}{x}-\frac{1}{x-\lambda}\right)\right] .
$$

The constant $C$ is evaluated from the causal boundary conditions at the bunched beam boundaries. As a simple example, for the wake-fields behind an infinitesi- 
mally short $\delta$-function beam of surface charge density $\sigma_{b}$ we have

$$
C=\left(\frac{\omega_{p} \sigma_{b}}{n_{0} c}\right)^{2}+x_{0}+\frac{1}{x_{0}}+\epsilon\left(1-\beta_{c 0}\right)\left(\frac{1}{x_{0}}-\frac{1}{x_{0}-\lambda}\right)
$$

where $x_{0}=\lambda+\left[\left(1-\beta_{c 0}\right) /\left(1+\beta_{c 0}\right)\right]^{1 / 2}$. The electric field is now known explicitly as a function of $x$, as $e E=\left(m_{e} c \omega_{p}\right) x^{\prime}$, and its maximum deceleration amplitude is $e E_{m}=\omega_{p} n_{0} \sigma_{b} / c$ directly behind the driving beam. The electric field also attains this maximum amplitude: in the wave's accelerating phase, when $x=x_{0}$ and $x^{\prime}=-x_{0}^{\prime}$

The solution to Eq. (10) can be written in terms of elliptical integrals, but this does not illuminate the behavior of the plasma waves or the continuous beam distribution well. However, one can deduce the prominent characteristics of the continuous beam loaded wake-fields from inspection of Eqs. (7) and (10). If the beam is relativistic and $\epsilon \ll 1$, then $x_{0} \simeq 1$ and $\lambda \simeq 1+\epsilon-1 / 2 \gamma_{c 0}$ where $\gamma_{c 0}=\left(1-\beta_{c 0}^{2}\right)^{1 / 2}$ is the initial continuous beam Lorentz factor. If the driving beam charge is large enough then the value of $x=x_{\min }$ at the density turning point in the forward motion of the plasma electrons can approach $\lambda$ from above. The continuous beam electrons build up a very high density near this point, as can be seen by rewriting Eq. (7) as $n_{c} / n_{0}=\epsilon\left(1-\beta_{c 0}\right)\left[1+1 /(x-\lambda)^{2}\right]$. This concentration of beam electrons is due to the wave potential's near trapping of the beam. As these electrons are accelerated to a very high energy $\gamma_{c} m_{e} c^{2}=$ $1 / 2\left(x_{c}+1 / x_{c}\right) m_{e} c^{2}=\simeq m_{e} c^{2} / 2\left(x_{\min }-\lambda\right)$, they load down the of asma wave and become detrapped and decelerated after attaining a high energy. This observation validates the assumption used in deriving the saturation of wave amplitude due to background trapping in Ref. 9. The continuous beam loading causes the wave 
electric field to turn faster, as can be seen from Eq. (10). For $\gamma_{c 0} \gg 1, \lambda \simeq 1$ and even small amplitude waves have nonlinear interactions with the continuous electron beam, as once $I \leq 1$ the last term in Eq. (10)becomes dominant and nonlinear. The local oscillation frequency increases dramatically in this case due to the sharp buildup of the continuous beam electrons. The wave period is shortened by this nonlinear effect, in analogy to the period shortening associated with thermal effects. ${ }^{|0|}$ The modulation of the continuous beam electron energy density corresponds to the excitation of a very nonlinear positive energy wave in the electron beam.

To illustrate the effects of beam modulation and period shortening we display a numerical example. A wave is assumed to be excited by a $\delta$-function beam with $\sigma_{b}=0.3 c n_{0} / \omega_{p}$, which gives an electric field amplitude of $e E_{m} / m c \omega_{p}=0.3$. The wave electric field and perturbed plasma density $n_{1} / n_{0}=n / n_{0}-1-\epsilon$, in absence of a continuous beam, shown in Fig. 1(a) are slightly nonlinear, as we have chosen a fairly large wave amplitude in which $n_{1} / n_{0} \simeq 0.3$ is not very small compared to unity. Upon introduction of continuous beam of initial speed $\beta_{c 0}=0.99\left(\gamma_{c 0} \simeq 7.7\right)$ and $\epsilon=0.1$, we observe the sharp peak of continuous electron beam density which serves to nearly completely suppress the region of positive perturbed plasma electron density, as illustrated in Fig. 1(b). The maximum continuous beam density reached is approximately $n_{c} \simeq 7.8 n_{0}$ (off scale), and the maximum energy $\gamma_{c} \simeq 62.5$. The net wave period has contracted to approximately 0.6 of the case in Fig. $1(\mathrm{a})$.

A physical criterion for strong buildup of continuous beam electrons can be formulated by comparing the surface charge density necessary to reverse the wave 
electric field to the integrated density of continuous beam electrons overtaken by the driving beam in the distance it takes a point in the continuous beam to slip one plasma period behind the driver. This gives a measure of the potential strength of the low energy beam's effect on the plasma wave. The criterion is thus

$$
\frac{\epsilon}{1-\beta_{c 0}} \geq \frac{e E_{m}}{m_{e} c \omega_{p}}
$$

This expression shows explicitly the requirement that the continuous beam be relativistic for significant interaction with the plasma wave to occur. If Eq. (12) is satisfied, then the continuous electron beam loading will cause a sudden reversal of the accelerating phase of the wave. The concommitant period shortening is pronounced in small amplitude waves, but not in very large amplitude waves, as the positive perturbed plasma electron density region is already very short and spiky in this case. ${ }^{\text {[6] }}$

It is of interest place these results in the context of plasm: 1 wave instability theory. The dispersion relation for linear waves in the beam-plasma system we have discussed is ${ }^{[1]}$

$$
\frac{\omega_{p}^{2}}{\omega^{2}}+\frac{\omega_{c}^{2}}{\left(\omega-k v_{c}\right)^{2}}=1
$$

where $\omega_{c}^{2}=4 \pi e^{2} n_{c} / \gamma_{c 0}^{3} m_{e}$. If we apply the additional wake-field excitation condition $\omega / k=v_{b}$ then the solution to Eq. (13) must give purely real value for the frequency and wave-number. Thus no absolutely unstable solutions which grow exponentially with the distance behind a causal excitation moving at speed $v_{b}$ can exist. The spatial and temporal characteristics of the two-stream instability are much more subtle, showing both absolute and convective nature. The Green's 
function response of a cold beam-plasma system has been found to be a growing wave-packet with frequency $\sim \omega_{p}$ and a group velocity two-thirds of the beam velocity $v_{c}{ }^{[12]}$

This result suggests that one might use the two-stream instability to ampiify plasma wake-fields. This is problematic for several reasons. The first is that energy in the instability can only propagate at best at two-thirds the speed of light, and thus wave energy obtained from the unstable beam can interact in only a limited way with accelerating relativistic particles. Also, since we wish to have a relativistic phase velocity in the wake-fields, and we want to extract energy from the low energy beam causally, we require the wake-field excitation to have a speed and $v_{b} \geq v_{c}$. Unfortunately, waves with phase velocity near $v_{c}$ have a very small nonlinear saturation level, as could be anticipated from our previous results. Thus the instability is not likely to be of value for wake-field amplification.

Once the effect of modulation of low energy electron beams in plasma wakefields has been experimentally achieved, it could be exploited as a source of high-frequency high-power fields. By passing the tightly bunched beam through an RF cavity one could extract efficiently extract the beam energy in the form of electromagnetic waves. Alternatively, a sudden lowering of the plasma density at the end of the interaction length would shift the peaks in the beam density inco a decelerating phase, and the wake-field amplitude would grow linearly with distance behind the driving beam, resulting in a potentially very large accelerating wake-field amplitude. In order for the modulation of a continuous low energy electron beam to be experimentally observed, the low energy beam must 
be stable in the interaction time against the two-stream instability. This limits the the plasma length to $L<(2 c / \sqrt{3})\left(2 / \omega_{c}^{2} \omega_{p}\right)^{1 / 3}$. One must allow enough time for the modulation to develop, however, and this implies approximately $L>m_{e} c^{2} /\left[e E_{m}\left(1-\beta_{c 0}\right)\right]$.

This work is supported by the U.S. Department of Energy, Division of High Energy Physics, Contract W-31-109-ENG-38. 


\section{REFERENCES}

1. P. Chen, J. M. Dawson, R. W. Huff and T. Katsouleas, Phys. Rev. Lett. 54,693 (1985).

2. T. Katsouleas, Phys, Rev. A 33, 2056 (1986).

3. S. Wilks, T. Katsouleas, J. M. Dawson, P. Chen, and J.J. Su, IEEE Trans. Plasma Sci., PS-15, 210 (1987).

4. J. B. Kosenzweig, P. Schoessow, B. Cole, W. Gai, R. Konecny, J. Norem, and J. Simpson, Argonne Preprint ANL-HEP-PR-88-43, submitted to Phys. Rev, Lett.

5. J. B. Rosenzweig, D. B. Cline, B. Cole, H. Figueroa, W. Gai, R. Konecny, J. Norem, J. Simpson, and P. Schoessow, Phys. Rev. Lett. 59, 98 (1988).

6. J. B. Rosenzweig Phys. Rev. Lett. 58 (1987) 555, and J. B. Rosenzweig IEEE Trans. Plasma Sci. PS-15 186 (1987).

7. R. D. Ruth, A. Chao, P. L. Morton, and P. B. Wilson, Particle Accelerators 17 (1985) 171.

8. A. Ts. Amatuni, S. S. Elbakian, and E. V. Sekhpossian, Yerevan Physics Institute Report No. ERFI 85-832, 1985 (to be published).

9. J. B. Rosenzweig, Proceedings of the 1987 Washington Particle Accelerator Conference, E. R. Lindstrom and L. S. Taylor, Editors, 124, IEEE, New York, (1987), and J. B. Rosenzweig, Argonne preprint ANL-HEP-PR-88-31 (1988) to be published in Phys. Rev. A. (October 1988)

10. T. Katsouleas and W. Mori, Phys. Rev. Lett., 61, 86 (1988). 
11. Richard J. Briggs, Electrons Stream Interaction With Plasmas, MIT Press, Cambridge, Mass., p. 48 (1964).

12. Michael E. Jones, Don S. Lemons, and Michael A. Mostrom, Phys. Fluids

; 26, 2784 (1983),

\section{FIGURE CAPTIONS}

Figure 1. Perturbed plasma electron density $n_{1}=n-n_{0}+n_{c 0}$, continuous beam density $n_{c}$ and electric field $E$, in wake-field plasma wave driven by $\delta$-function beam with $\sigma_{b}=0.3 e n_{0} / \omega_{p}\left(e E_{m} / m c \omega_{p}=0.3\right)$. Densities normalized to $n_{0}$, electric field normalized to $m c \omega_{p} / e$. (a) No continuous beam. (b) Continuous beam with $\epsilon=0.1$ and $\beta_{c 0}=0.99$. Maximum beam density $n_{c} \simeq 7.8 n_{0}$ (off scale). 


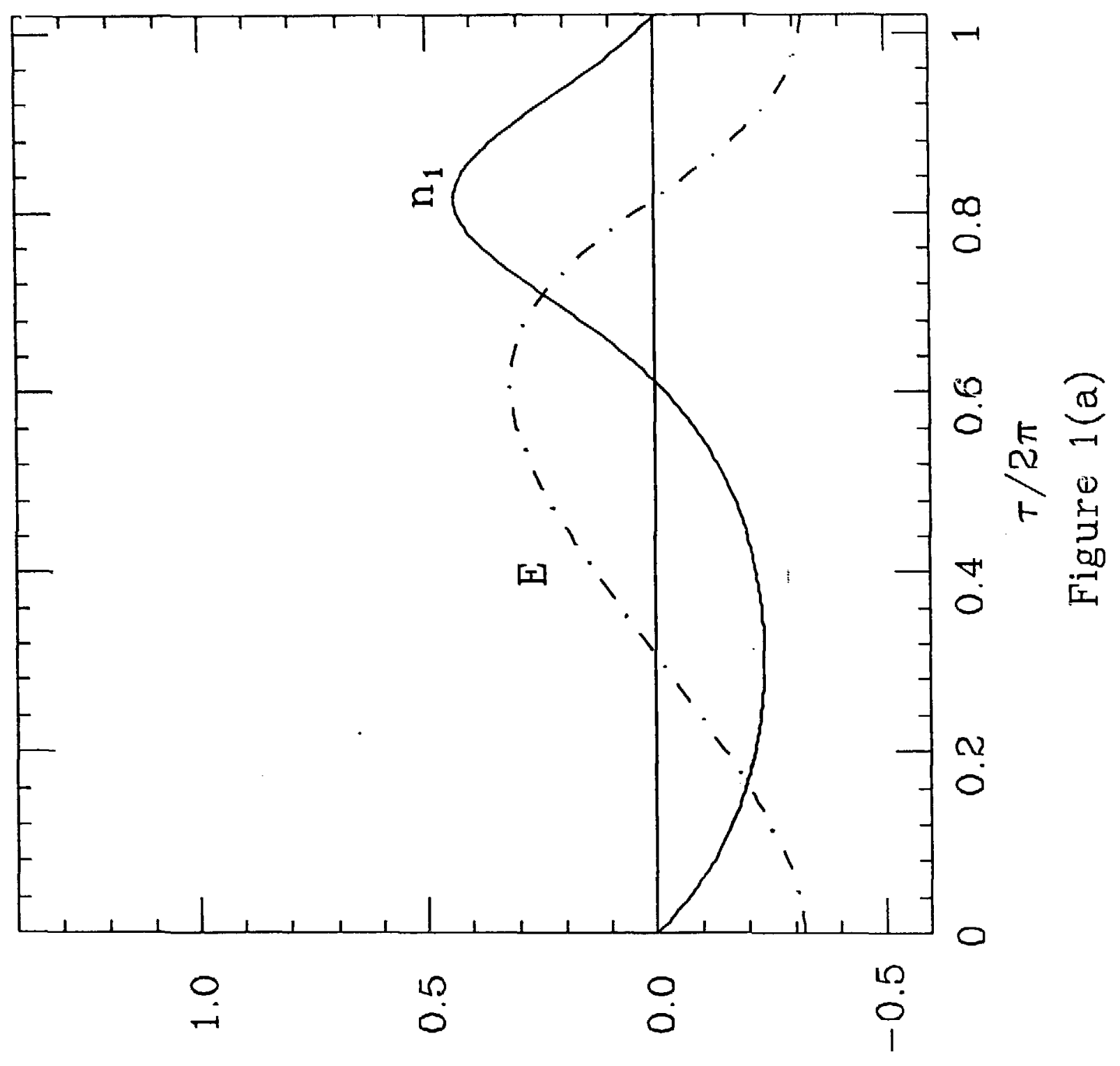




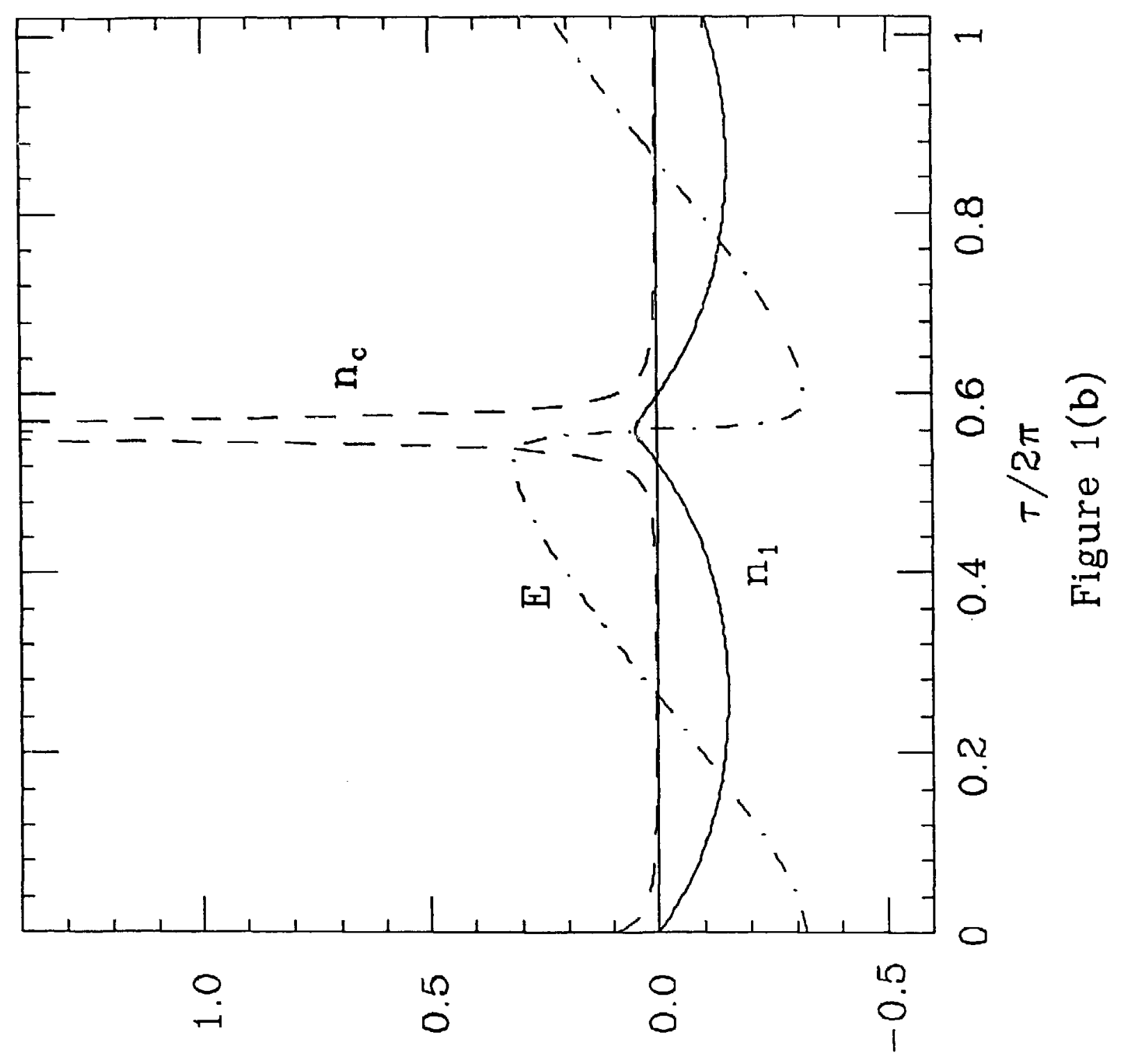

